\title{
Cellotape Birefringent Filter: Some New Demonstrations
}

\author{
Ayantika Khanra, Barun Raychaudhuri* \\ Department of Physics, Presidency University, Kolkata, India \\ Email: "barun.raychaudhuri@gmail.com
}

Received 29 May 2016; accepted 4 July 2016; published 7 July 2016

Copyright (c) 2016 by authors and Scientific Research Publishing Inc.

This work is licensed under the Creative Commons Attribution International License (CC BY). http://creativecommons.org/licenses/by/4.0/

(c) †) Open Access

\begin{abstract}
An easy method is put forward to estimate the optical bandwidth and the wavelength of maximum transmission of Lyot optical filter by measuring the change in phase retardation of birefringent plates as function of thickness. The objective is to demonstrate the experiment with common undergraduate laboratory equipment and thereby provide with an educational aid. The filter is fabricated with cellotape using its birefringence property. The accuracy of measurement is crosschecked with precision spectroradiometric measurements. Some simplification is suggested in the theoretical derivation of the transmitted intensity and a possibility of realizing tunable filter by changing the angle of incidence is indicated.
\end{abstract}

\section{Keywords}

Cellotape, Birefringence, Lyot Filter

\section{Introduction}

Colored light can be obtained from white light by passing it through selectively absorbing materials like glass filter, by dispersion through prism and by diffraction through grating. Another method of producing monochromatic light is to use the birefringence property of materials [1]. The principle is that the polarized light transmitting normally through a birefringent plate termed as retarder splits into two orthogonal linear waves having a relative phase difference subject to the birefringence property of the plate. Depending on the phase difference, waves of different wavelengths may cancel out or get reinforced. Thus wavelength selectivity is achieved. Only the components parallel to the transmission axis of another polarizer, termed as analyzer reach the observer. Such filters can offer narrow bandwidth and wide field of view [2] and also tuning capability [3]. Common birefringent materials are quartz, calcite and others like cadmium sulphide [2]. However, the use of commercial

${ }^{*}$ Corresponding author.

How to cite this paper: Khanra, A. and Raychaudhuri, B. (2016) Cellotape Birefringent Filter: Some New Demonstrations. Optics and Photonics Journal, 6, 139-145. http://dx.doi.org/10.4236/opj.2016.67016 
cellotape or scotch tape as an efficient and easily available birefringent material, at least for demonstration purpose has a long legacy [4]-[7]. Fabrication of Lyot filter [4] [7] and Solc filter [5] and transmission of a particular waveband of visible region are reported. Intensity variations of transmitted light with the orientations of the transmission axis of the analyzer and the optical axis of the retarder are investigated [6]. The property of rotating the direction of polarization of cellotape is demonstrated with interferometry [8]. The birefringence property of cellophane is used to rotate the polarization of a beam [9]. Obviously part of the common objectives of such studies was to build up a birefringent filter for demonstration purpose using handy, low-cost materials and to conduct the experiment in a common undergraduate laboratory.

The present work supports the above idea and puts forward a befitting measuring technique for such filter. Some simplification in the theoretical derivation is also suggested and the possibility of realizing tunable filter by changing the angle of incidence is indicated. A Lyot optical filter is fabricated using pieces of mobile phone screen as polarizers. The birefringent plates of different thicknesses are prepared by fixing counted layers of cellotape on glass slides. A method is suggested to estimate the full width half maximum (FWHM) of the filter by measuring the change in phase shift between the orthogonally polarized waves introduced by the birefringent plates of varying thickness. The proposed method worked well with reasonable accuracy, as cross-checked with precision spectroradiometric measurements.

\section{Background Theory}

\subsection{Principle of Lyot Filter}

This filter is comprised of a series of polarizers with pass axes in the same direction and having a birefringent plate of variable thickness inserted between each two polarizer. The plate thickness varies as $t, 2 t, 4 t, \cdots, 2^{N-1} t$ for $N$ number of plates, $t$ being the minimum thickness. The optic axes of all the plates are aligned at $45^{\circ}$ with respect to the aligned pass axes of the polarizers. The transmittance of light of wavelength $\lambda$ through the above filter is given by [2]

$$
T=\left(\frac{\sin 2^{N} \Gamma / 2}{2^{N} \sin \Gamma / 2}\right)^{2}
$$

where

$$
\Gamma=\frac{2 \pi}{\lambda} \Delta n t
$$

is the phase difference between the orthogonally polarized waves, henceforth termed as 'phase retardation', introduced by the birefringent plate of thickness $t$ and $\Delta n$ is the difference in refractive index of the plate for the two wave components.

The maximum transmittance through the filter occurs when $\Gamma=2 k \pi$, where $k=1,2, \cdots$, etc. and the corresponding wavelength for maximum transmission $\left(\lambda_{m}\right)$ assessed from Equation (2) is expressed as

$$
\lambda_{m}=\frac{\Delta n t}{k}, k=1,2, \cdots, \text { etc. }
$$

The bandwidth of the filter, estimated in terms of full width half maximum (FWHM) is determined by bandwidth of the thickest plate, multiplied by the factor 0.886 due to presence of other stages.

$$
\mathrm{FWHM}=\frac{0.886 \lambda_{m}^{2}}{2^{N} \Delta n t}
$$

which indicates that the bandwidth decreases and the filter becomes sharper, as the number of plates $(N)$ increases. However, a compromise is required because increased number of polarizers and retarders also diminishes the transmitted light intensity.

\subsection{Birefringence Determination}

The following methodology is proposed for determining the parameter $\Delta n$ for Equation (4) using Equation (2). The electric field vector of the monochromatic polarized light transmitted normally through a retarder can be 
represented as

$$
\boldsymbol{E}=E_{0} \cos \varphi \exp [i(\omega t+k z+\Gamma)] \hat{x}-E_{0} \sin \varphi \exp [i(\omega t+k z)] \hat{y}
$$

where $E_{0}$ is the initial amplitude, $z$ is the direction of light propagation, $x^{\prime}$ is the direction of polarizer transmission axis so that $\hat{x}$ and $\hat{y}$ represent the directions of the slow and fast axes, respectively, of the retarder. The arrangement is indicated in Figure 1. The angle between $x^{\prime}$-axis and the slow axis of the retarder is $\varphi$ and the phase retardation between the $x$ and $y$ component of the electric field vector is $\Gamma$; already defined with Equation (2). The other symbols have the usual meaning.

When this electric vector passes through the analyzer making angle $\theta$ with the $x^{\prime}$-direction, the $x$ and $y$ components make angle $(\theta-\varphi)$ and $\left(90^{\circ}+\varphi-\theta\right)$, respectively with analyzer pass axis. The emergent electric field has magnitude

$$
E_{1}=E_{0} \exp [i(w t+k z)][\cos \varphi \cos (\theta-\varphi) \exp (i \Gamma)-\sin \varphi \sin (\theta-\varphi)]
$$

so that the intensity (I) of the emergent light can be expressed, in terms of $E_{1}$ and its complex conjugate ( $E_{1}^{*}$ ) as $E_{1} E_{1}^{*} / 2$ and it can be derived algebraically that

$$
I=\frac{I_{0}}{2}\left[1+\left(\cos ^{2} 2 \varphi+\cos \Gamma \sin ^{2} 2 \varphi\right) \cos 2 \theta+\sin 2 \theta \sin 4 \varphi \sin ^{2}(\Gamma / 2)\right]
$$

where $I_{0}=E_{0}^{2} / 2$. When $\varphi=45^{\circ}$, Equation (7) reduces to

$$
I=\frac{I_{0}}{2}[1+\cos \Gamma \cos 2 \theta]
$$

It may be noted that Equation (8) is derived by algebraic simplification only, which is much easier. The same expression was derived earlier by Jones matrix method [6]. Equation (8) indicates that the experimental $I-\cos 2 \theta$ graph for the transmitted light intensity $(I)$ of known wavelength $(\lambda)$ measured for different analyzer angle $(\theta)$ keeping the optic axis of the retarder at $45^{\circ}$ with respect to polarizer pass axis should yield a straight line with slope of $\left(I_{0} / 2\right) \cos \Gamma$ and $I$-axis intercept of $I_{0} / 2$ and the value of $\Gamma$ can be determined for any arbitrary thickness $(t)$ of the birefringent plate.

\section{Experiments and Results}

The birefringent plates were fabricated by fixing commercial cellotape on thin glass slides. The thickness of a single film of cellotape was determined mechanically by measuring the average thickness of the full pack with micrometer of least count $10 \mu \mathrm{m}$ and then exfoliating it layer by layer and counting the number of layers. The average thickness $(t)$ of a single layer was found to be $38 \mu \mathrm{m}$. The thickness of the glass slide was immaterial because glass has no birefringence and the same glass was used everywhere so that its transmittance always acted as a constant term. Sodium vapor lamp was used as monochromatic source for determining $\Gamma$ from Equation (8). The transmitted light intensity was measured with Metravi 1330 digital light intensity meter calibrated in lux. Standard polarizer and analyzer graduated up to $2^{\circ}$ angular resolution were employed. It was verified that

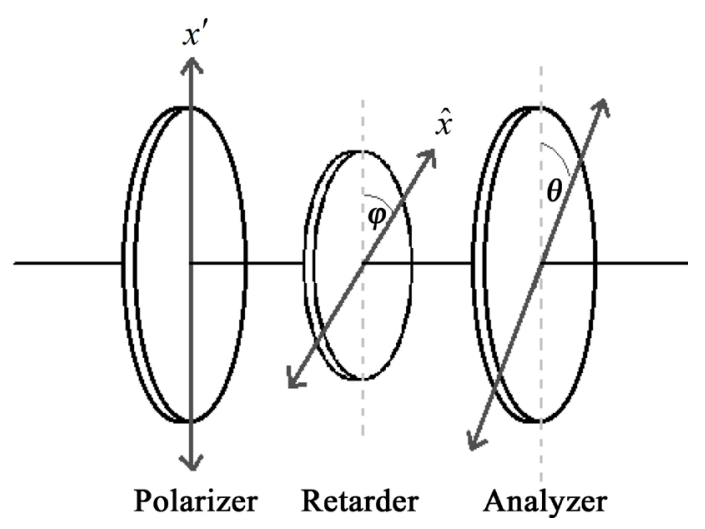

Figure 1. Diagram indicating direction of polarizer, analyzer and retarder axes. 
the blank glass slide did not introduce any polarization effect.

For fabricating the Lyot filter, $N=3$ was maintained. The architecture of the device is sketched in Figure 2. Since the thickness of a single layer of cellotape was too small $(38 \mu \mathrm{m})$, the resultant transmission might shift at ultraviolet region, as obvious from Equation (3). In order to keep the transmission within visible region, the thickness was multiplied by three and the retarder plates were prepared with three, six and twelve cellotape layers, respectively on glass slides. This time the polarizers were those extracted from mobile phone LCD screen. Tungsten-halogen lamp was used as white light source. The wavelength of maximum transmission $\left(\lambda_{m}\right)$ was determined with conventional spectrometer and grating arrangement, as mentioned in Table 1 . The following methodology was put forward to determine FWHM, the other filter parameter.

Equation (2) indicates that $\Gamma-t$ graph for any arbitrary thickness of retarder should be straight line with slope of $(2 \pi / \lambda) \Delta n$. The number of cellotape layers was increased from 1 to 10 so that the thickness of the retarder increased as multiples of $t$, the thickness of a single layer. In each case, $\Gamma$ was determined from Equation (8). The experimental variation of $\Gamma$ with thickness of cellotape layers is shown in Figure 3. The plot is fairly linear. Knowing $\lambda$, the birefringent parameter $\Delta n$ was determined from the slope. The numerical value of $\Delta n$, as calculated from the slope using Equation (2) was 0.00921 assuming the wavelength of sodium light $589.3 \mathrm{~nm}$. The fabricated lyot filter transmits green light, and it was assumed $\Delta n$ for green light and $\Delta n$ measured at $589.3 \mathrm{~nm}$ is approximately same. Knowing $N, \Delta n, \lambda_{m}$ and $t$, FWHM was determined using Equation (4) and compiled in Table 1. It also contains the value of $\lambda_{m}$ calculated from Equation (3) using experimental value of $\Delta n$. Accurate determination of $t$ is essential because even $5 \%$ deviation can lead to wide change in $\lambda_{m}$, as mentioned in Table 1 . The requirement is satisfied here because the micrometer least count $(10 \mu \mathrm{m})$ is much less than $t(=38 \times 3 \mu \mathrm{m})$.

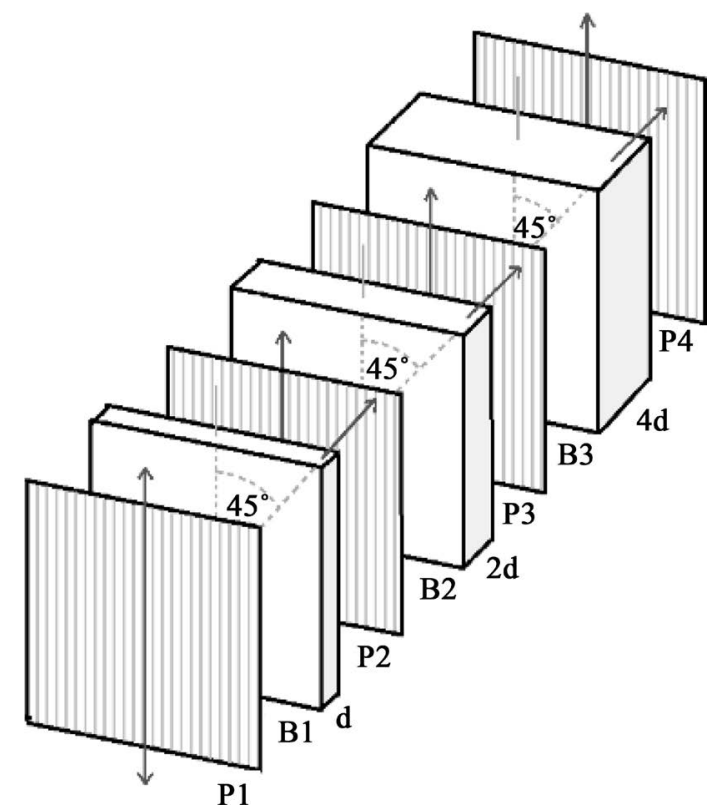

Figure 2. Architecture of three-stage Lyot filter: $P_{1}, P_{2}, P_{3}$ and $P_{4}$ are the polarizers with vertical pass axes and $B_{1}, B_{2}$ and $B_{3}$ are the three birefrinent plates of thickness $d, 2 d$ and $4 d$, respectively having optic axes inclined at $45^{\circ}$ with respect to the polarizer pass axes.

Table 1. Comparison of Lyot filter parameters determined by present method and spectroradiometer measurements.

\begin{tabular}{clc}
\hline \multicolumn{1}{c}{ Parameter } & \multicolumn{1}{c}{ Present Method } & Spectroradiometric data \\
\hline & $\begin{array}{l}\text { (i) measured with spectrometer \& grating: } 529-532 \\
\text { (ii) Calculated from Equation (3) with experimental } t \text { and } \Delta n \text { and using } k=2:\end{array}$ & $532-534$ \\
$\lambda_{m}(\mathrm{~nm})$ & $\begin{array}{l}\text { (a) } 525 \text { with exact value of } t \\
\text { (b) } 498 \text { assuming } 5 \% \text { increase in } t \\
\text { (c) } 551 \text { assuming } 5 \% \text { decrease in } t\end{array}$ \\
FWHM (nm) & 28 (calculated from Equation (3) with experimental $\Delta n$ ) & 30 \\
\hline
\end{tabular}


In order to check the accuracy of the above method, the spectrum of the transmitted light of the tungstenhalogen source passing through the filter was directly measured with accurate Analytical Spectral Devices spectroradiometer, which can record the relative intensities of radiation at almost $1 \mathrm{~nm}$ resolution. The parameters $\lambda_{m}$ and FWHM were directly measured and compared with the above results, as mentioned in Table 1 . The possible deviation may be due to the fluctuation in cellotape thickness. It is indicated in Table 1 how sensitive the wavelength is with the thickness.

Theoretical variation of transmittance with wavelength for the Lyot filter was generated, as shown in Figure 4 by putting the above mentioned experimental value of $\Delta n$ in Equation (2) and using Equation (1). A similar spectrum was experimentally obtained with spectroradiometer for the fabricated Lyot filter, as displayed in Figure 5. It shows both the directly measured spectrum for the transmitted light (Figure 5(a)) and the relative spectrum obtained by dividing it by the emission spectrum of the original white light for each wavelength (Figure 5(b)). All the spectral curves are normalized with respect to their respective maximum values. Comparing Figure 4 and Figure 5 it is apparent that the nature of wavelength variation of the transmittance of the filter is similar to that predicted in Figure 4. Unlike the theoretical curve of Figure 4, the transmission peaks in the experimental curves are not of the same height for visible and infrared regions. It is so because the experimental white light does not have uniform intensity in spectrum throughout the wavelength range. The transmission of the fabricated filter does not match with the theory in the infrared region and it does not work in infrared region. A possible reason may be the absorption of infrared through the glass substrate. However, the device works very well in the visible region of wavelength. The values of $\lambda_{m}$ and FWHM determined by the present method and from spectroradiometer data are in good agreement and also match the predicted value, as compared in Table 1.

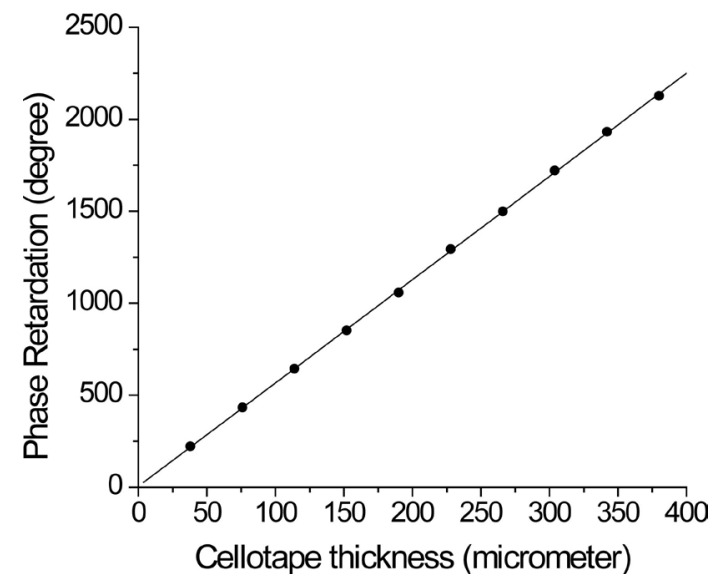

Figure 3. Experimental variation of retardance $(\Gamma)$ with cellotape thickness.

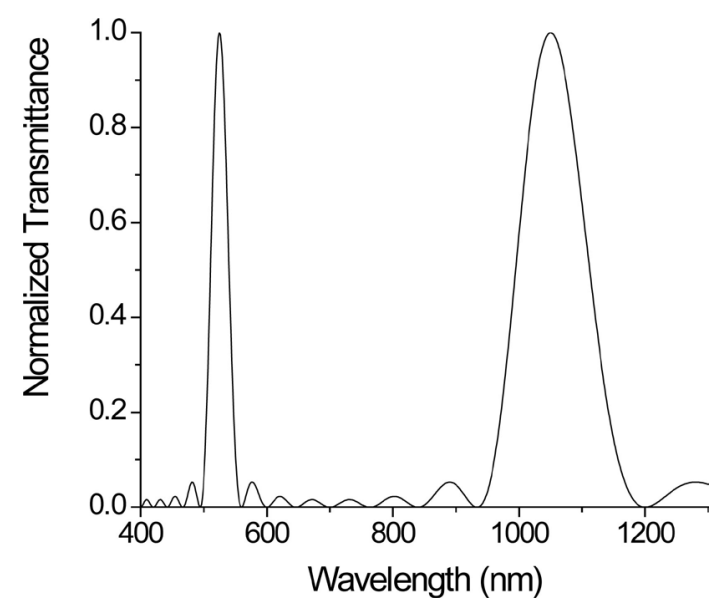

Figure 4. Theoretical variation of transmission with wavelength for three-stage Lyot filter. Parameters: $N=3, \Delta n=0.00921, d=114 \mu \mathrm{m}$. 
The relative transmission of the filter was also studied with four different light sources of distinct wavelengths, namely sodium lamp and semiconductor lasers of red, green and blue colours. The transmission percentages compiled in Table 2 indicate the efficient wavelength selectivity of the fabricated filter. Indeed the wavelength selectivity was the main subject of this work and normal incidence of light, i.e. angle of incidence $0^{\circ}$ was considered in the above cases. However, a possibility of wavelength tuning by changing the angle of incidence is also indicated in Figure 6. At least in the case of visible wavelength, the change of the peak transmission wavelength with angle of incidence is quite prominent. For $0^{\circ}, 15^{\circ}$ and $25^{\circ}$, the corresponding peak transmission wavelengths were found to be $532 \mathrm{~nm}, 523 \mathrm{~nm}$ and $505 \mathrm{~nm}$, respectively.

\section{Conclusion}

The present work demonstrates an experiment comprising the fabrication of Lyot birefringence filter and measurement of its wavelength selectivity; all carried out within common undergraduate laboratory environment. The Lyot birefringent filter is fabricated with mobile phone screens as polarizers and cellotape layers on glass

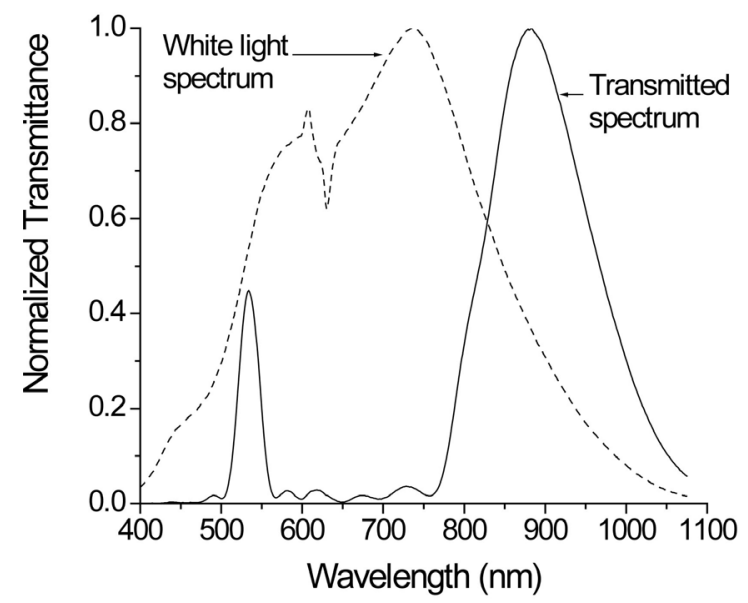

(a)

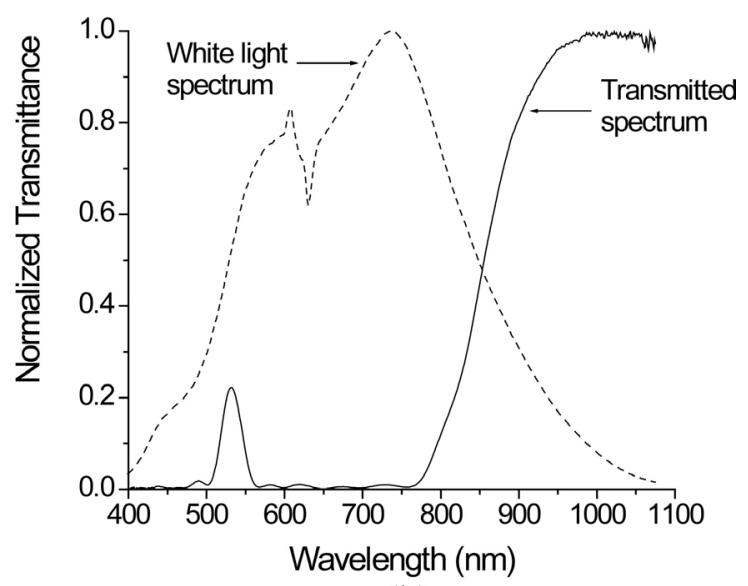

(b)

Figure 5. Experimental transmission spectrum for the fabricated three-stage Lyot filter (solid line) both (a) directly measured and (b) divided by the actual spectrum of the white light source indicated by dotted lines.

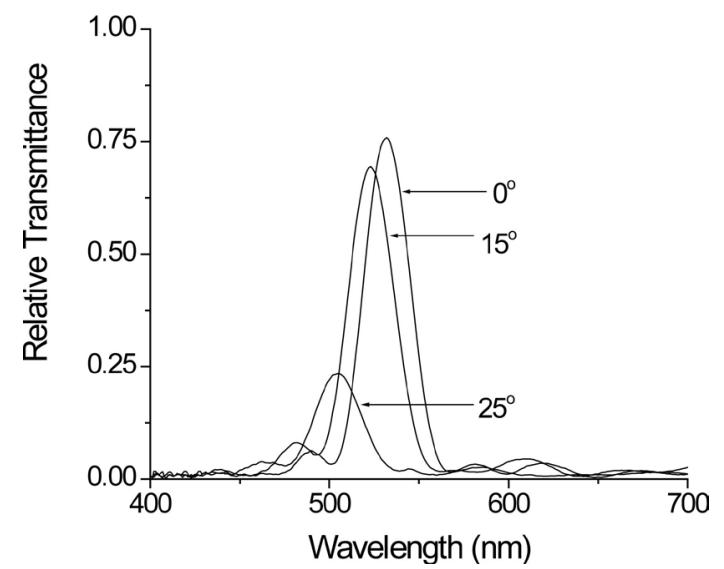

Figure 6. Tuning of the filter by changing the angle of incidence mentioned against the curves.

Table 2. Comparison of transmission of light from different sources through the Lyot filter.

\begin{tabular}{ccccc}
\hline Source & Blue laser & Green laser & Sodium light & Red laser \\
\hline Transmission (\%) & 0.36 & 22.0 & 8.0 & 0.20 \\
\hline
\end{tabular}


slides as birefringent plates. The basic theory of birefringence is discussed and an equation relating the transmitted intensity to the analyzer and plate orientations is derived by algebraic method. An easy method is put forward to estimate the full width half maximum (FWHM) of the filter by measuring the change in phase retardation introduced by the birefringent plates of varying thickness. The correctness of the results obtained by the proposed method is assured by comparing with those obtained from precision measurements with spectroradiometer. The expression for the transmitted intensity is derived only by algebraic simplification. Also the possibility of realizing tunable filter by changing the angle of incidence is suggested.

\title{
Acknowledgements
}

The authors acknowledge the facilities of using the equipment and other infrastructural conveniences obtained from the laboratories of the Department of Physics, Presidency University.

\section{References}

[1] Hecht, E. (2002) Optics. 4th Edition, Addision Wesley, San Francisco, 358-360.

[2] Yeh, P. (1981) Dispersive Birefringent Filters. Optics Communications, 37, 153-158. http://dx.doi.org/10.1016/0030-4018(81)90407-7

[3] Buhrer, C.F. (1994) Synthesis and Tuning of High-Order Solc-Type Birefringent Filters. Applied Optics, 33, 22492254. http://dx.doi.org/10.1364/AO.33.002249

[4] Cloud, S.D. (1973) Birefringence Experiments for the Introductory Physics Course. American Journal of Physics, 41, 1184-1188. http://dx.doi.org/10.1119/1.1987511

[5] Velasquez, P., Sanchez-Lopez, M.M., Moreno, I., Puerto, D. and Mateos, F. (2005) Interference Birefringent Filters Fabricated with Low Cost Commercial Polymers. American Journal of Physics, 73, 357-361. http://dx.doi.org/10.1119/1.1834920

[6] Belendez, A., Fernandez, E., Frances, J. and Neipp, C. (2010) Birefringence of Cellotape: Jones Representation and Experimental Analysis. European Journal of Physics, 31, 551-561. http://dx.doi.org/10.1088/0143-0807/31/3/012

[7] Gluck, P. and King, J. (2014) Birefringence in Cellulose Tapes: Chapter-39 of Physics Project Lab. Oxford University Press, Oxford, 207-211.

[8] Kinyua, D.M., Rurimo, G.K., Karimi, P.M., Maina, S.N. and Ominde, C.F. (2013) Interferometry Analysis of Cellophane Birefringence. Optics and Photonics Journal, 3, 337-341. http://dx.doi.org/10.4236/opj.2013.36052

[9] Kalwe, J., Neugebauer, M., Ominde, C., Leuchs, G., Rurimo, G. and Banzer, P. (2015) Exploiting Cellophane Birefringence to Generate Radially and Azimuthally Polarized Vector Beams. European Journal of Physics, 36, 025011. http://dx.doi.org/10.1088/0143-0807/36/2/025011

\section{Scientific Research Publishing}

\section{Submit or recommend next manuscript to SCIRP and we will provide best service for you:}

\author{
Accepting pre-submission inquiries through Email, Facebook, Linkedin, Twitter, etc \\ A wide selection of journals (inclusive of 9 subjects, more than 200 journals) \\ Providing a 24-hour high-quality service \\ User-friendly online submission system \\ Fair and swift peer-review system \\ Efficient typesetting and proofreading procedure \\ Display of the result of downloads and visits, as well as the number of cited articles \\ Maximum dissemination of your research work
}

Submit your manuscript at: http://papersubmission.scirp.org/ 